\title{
Contact mechanics of pad of grasshopper (Insecta: ORTHOPTERA) by finite element methods
}

\author{
DAI ZhenDong ${ }^{1 \dagger} \&$ GORB Stanislav ${ }^{2 \dagger}$ \\ ${ }^{1}$ Institute of Bio-inspired Structure and Surface Engineering, Nanjing University of Aeronautics and Astronautics, Nanjing, Jiangsu, \\ China 210016; \\ ${ }^{2}$ Evolutionary Biomaterials Group, Max-Planck-Institute for Metals Research, Germany, 70569
}

During locomotion, insect feet endure dramatic impact force and generate adhesive force which is controlled by the architecture of the foot. The patterns of smooth attachment pads in insect feet vary widely among insect orders and families. The functional significance of the micro-structure and geometric design of insect feet remains largely unknown. In this study, we developed a two-dimensional finite element model of a grasshopper's attachment pad. Realistic geometric microstructure and material properties are applied in the biomechanical analysis of the structural behavior during contact. Here we use scanning electronic microscopy to study the microstructure of the grasshopper's pad, and then use the finite element method to calculate the deformation vector fields, contact stiffness, contact area, function of the airbag and strain fields during the contact process. The results reveal that the geometric design and material topology of a grasshopper's pads are very effective in reducing contact stiffness, increasing contact area and generating high friction force during the contact procedure. The rod-containing structure supporting the soft exocuticle makes the pads highly adaptive to various surfaces and decreases the stress inside the pads.

grasshopper, contact mechanics, functional morphology, biomimetics, finite-element analysis

The foot is one of the key organs for both animals and legged vehicles. The mechanical interactions between the foot and substrate are extremely complex. Efficient and reliable locomotions-running, jumping, landing and even moving on up-side-down surfaces-depends on the efficiency of the foot, which, in turn, comes from its structure and control. Analysis of the relationship between the function and structure of insect feet helps us to get a better understanding of their over millions of years evolution and to face the challenges of life in an uncertain and changing environment ${ }^{[1,2]}$. The biomechanics of the human foot has been studied to improve sport shoe design and make clear the effects of Diabetes mellitus on foot function ${ }^{[3]}$, and finite elements analysis (FEA) has been employed to reveal the biomechanical characteristics of human feet ${ }^{[4-6]}$ and the effects of Diabetes mellitus ${ }^{[7,8]}$. The FEA has been applied to the functional morphology of the foot by three basic steps in the research ${ }^{[9]}$. FEA has also been employed to create a three-dimensional finite element model of a horse's foot $^{[10]}$. Insects have even more remarkable locomotion abilities than humans and horses. Grasshoppers can fly, jump, walk and run on various surfaces. The biomechanical characteristics of dragonfly wings and the fly's wing structure and deformation during flight ${ }^{[11]}$ and the function of the hind wing in the desert locust ${ }^{[12,13]}$ have also been studied using FEA. But until now, FEA has not been used to analyze the biomechanical aspects of insect attachment pads.

Artificial legged vehicles, especially three-dimen-

\footnotetext{
Received July 29, 2008; accepted October 14, 2008

doi: 10.1007/s11434-009-0088-4

†Corresponding author (email: zddai@nuaa.edu.cn; s-gorb@mf.mpg.de)

Supported by the National Natural Science Foundation of China (Grants Nos. 60535020, 50635030 and 50675160) and the Development Plan of the State Key Fundamental Research (Grant No. 2007CB607600)
} 
sional obstacle-free (TDOF) robots, such as the geckolike robot, have been attracting great attention in recent years for their terrain-adaptive ability and possible applications in hazardous and extraterrestrial environments. Adhesive feet serve to generate attractive force between pads and contacted surfaces to avoid the robot overturning or falling down when it moves on walls or ceilings. Foot design is still a key problem for TDOF robots, and techniques in their design still lag far behind requirements ${ }^{[14]}$. Foot design is a key technology for developing biped robots because of the importance of the foot in increasing stability and decreasing impact force when contacting target surfaces ${ }^{[15]}$. It has been reported that higher stiffness in the foot-leg system of legged robots can lead to failure in transmission gears because of the impact force during contact ${ }^{[16]}$.

It was suggested that the architecture of insects' adhesive feet can be categorized into smooth soft pads and hairy pads ${ }^{[17]}$. Adhesion has been measured and a contact model based on Hertz theory has been proposed ${ }^{[18]}$. The JKR model has been used to explain the differences in smooth attachment pads ${ }^{[19,20]}$. The anisotropy in frictional forces and secretion from pads have been noted $^{[1,21,22]}$. Microstructure studies using SEM and histological anatomy show that the material along the contact part in grasshopper's foot, the exocuticle, is a highly deformable material, while the hard black cuticle contains hemolymph and an airbag ${ }^{[1]}$. Neither the Hertz theory nor the JKR model can be accurately applied to this type of geometric structure. It has been proposed that Van der Waals forces are the adhesive mechanism for hairy attachment pads ${ }^{[23-25]}$, and the effects of mi- cro-suckers $^{[26]}$ and static electricity ${ }^{[27]}$ have also been investigated.

It has been proposed that the adhesive mechanism for smooth attachment pads, such as locust's and grasshopper's pads, is a capillary force For this mechanism the actual contact area is a major factor for adhesion force. The effect of the geometric design of the grasshopper's foot on its contact parameters not only helps explain its evolution, but also provides assistance to robot designers to create better feet for legged robots. Here, we study the contact mechanics of a grasshopper (Insecta: ORTHOPTERA) foot on a hard smooth surface using finite element method. The goal of this investigation is to help understand why the grasshopper foot evolved to its present geometric form from a biomechanical point of view, and how its material parameters and topology influence foot deformation and adhesion.

\section{Materials and methods}

\subsection{Geometric model and mechanical parameters}

Figure 1(a) is a photo of a grasshopper attachment pad (foot) in the long hind leg, which consists of 4 tarsomere (T1-T4) of which the first three have smooth attachment pads (euplantulae) and the 4th is claw (T4). Figure 1(b) shows a diagram of a cross-section in the third tarsomere, where AS is the airbag; HM is the hemolymph; $\mathrm{HK}$ is the hard cuticle; TD is the flexor tendon of the claw. Figure 1(c) shows the microstructures of surface and sub-surface on attachment pads. EXO is the exocuticle that contains a rod based structure supporting the superficial layer and CL is the epidermal cells. The sur-
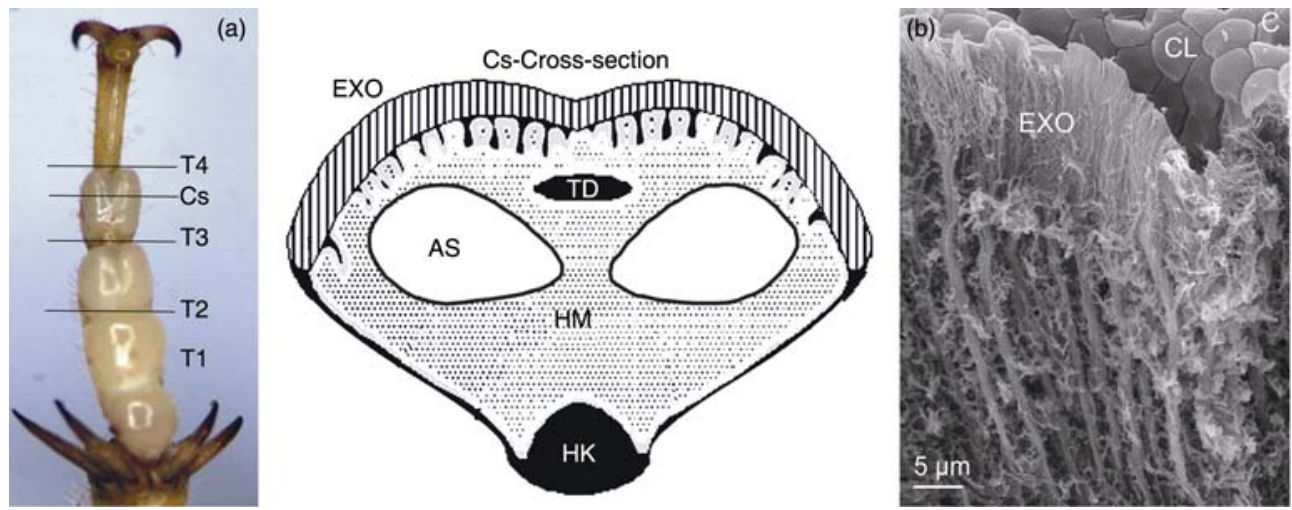

Figure 1 Grasshopper's attachment pads (a) and an illustration of the cross section (b) on 3rd tarsmere. (a) Tarsus includes tarsomeres (T1-T3) with attachment pads and claw (T4); Cs-cross section; (b) Diagram of cross-section of third tarsomere. AS-airbag; HMhemolymph; $\mathrm{CL}$-epidermal cells; $\mathrm{HK}$-hard cuticle; TD-flexor tendon of claw; EXO-exocuticle with rods structure supporting superficial layer. 
face has a hexagonal pattern when observed under SEM. The microstructure of the pads is presented into 2 layers: a superficial density soft layer EXO $(6-10 \mu \mathrm{m})$ and under the EXO are the grove-like structures. The rods are $2-3 \mu \mathrm{m}$ in diameter and $40-50 \mu \mathrm{m}$ in length which are chitin filaments and are perpendicular to the primary surface ${ }^{[1,19]}$.

To model the pad for FEA, we change the elastic modulus of the fluid in the area surrounded by EXO and HK to simulate the effect of air pressure in airbag. The effect of the flexor tendon (TD) is excluded, because it drives the movement of claw and tarsus and its function cannot be reflected in a two-dimensional model. Figure 1(b) was obtained by inputting an SEM image of the cross-section into drawing software (CorelDraw 11) and the geometric model created by fitting various boundaries. The cross-section of the third tarsomere was simplified into three parts - the soft layer exocuticle (EXO), the hard cuticle (HK) and the liquid-filled container. This model is named the 'fluid-contained' model (FC). For comparison, we set up another model only consisting of the EXO and $\mathrm{HK}$, named the solid soft-material model (SS). The two models are shown in Figure 2(a) and (b), respectively. We used ANSYS 5.6 for the finite element analysis.

The elastic modules of the biomaterials EXO and HK quoted from ref. [28] ref. [29] are $\mathrm{E}_{\mathrm{EXO}}=74.2 \mathrm{~N} / \mathrm{mm}^{2}$ and $\mathrm{E}_{\mathrm{HK}}=3775 \pm 73 \mathrm{~N} / \mathrm{mm}^{2}$. EXO is a macro-deformed material, and we its Poisson's ratio was chosen as 0.45 , similar to that of rubber. The HK's Poisson's ratio was chosen to be 0.3. The elastic modulus for the liquid integrated from hemolymph and airbag was set at $\mathrm{E}_{\mathrm{F}}=$
$2436.6 \mathrm{~N} / \mathrm{mm}^{2}$ (water's value) and density $=10^{-6} \mathrm{~kg} / \mathrm{mm}^{3}$ because hemolymph consists of $92 \%$ water. No elastic modulus was measured. The elastic modulus of the target surface, i.e. glass $\mathrm{E}=55000 \mathrm{~N} / \mathrm{mm}^{2}$, is much higher than the EXO and HK, so it is treated as a rigid body.

\subsection{Elements and mesh}

We introduced plan model to present the contact mechanics of grasshopper's attachment pads to the target surface. The contact target surface is a glass plane and defined as a rigid body in the model. The EXO is considered a twodimensional hyper-elastic solid (HYPER56). The element used to represent the HK was a two-dimensional 8 node structured solid (PLANE82). The two-dimensional contained element (Fluid79) represents the fluid. The two-dimensional general contact elements (CONTACT48) represent the contact.

\subsection{Boundary conditions and loads}

The target surface TS was fully restrained $\Delta X=\Delta Y=0$. Displacement on the axis of symmetry $Y$ was zero and set at $\Delta X=0$ because the structure is symmetrical and the load is parallel to the symmetry axis. No direct measurements on the ground reaction forces of grasshopper's hind leg were carried out. The reaction force, around tens to hundreds micro Newton, was estimated by studying the jumping energy ${ }^{[28,29]}$. The preloading displacements were given by a consideration to cover the range of jumping force. Here the loads were set up as restrained displacement on the HK surface from 0.01 to $0.1 \mathrm{~mm}$ (See Figure 2 for more details). The estimation was obtained under the presumption that the jump was performed in micro-seconds.
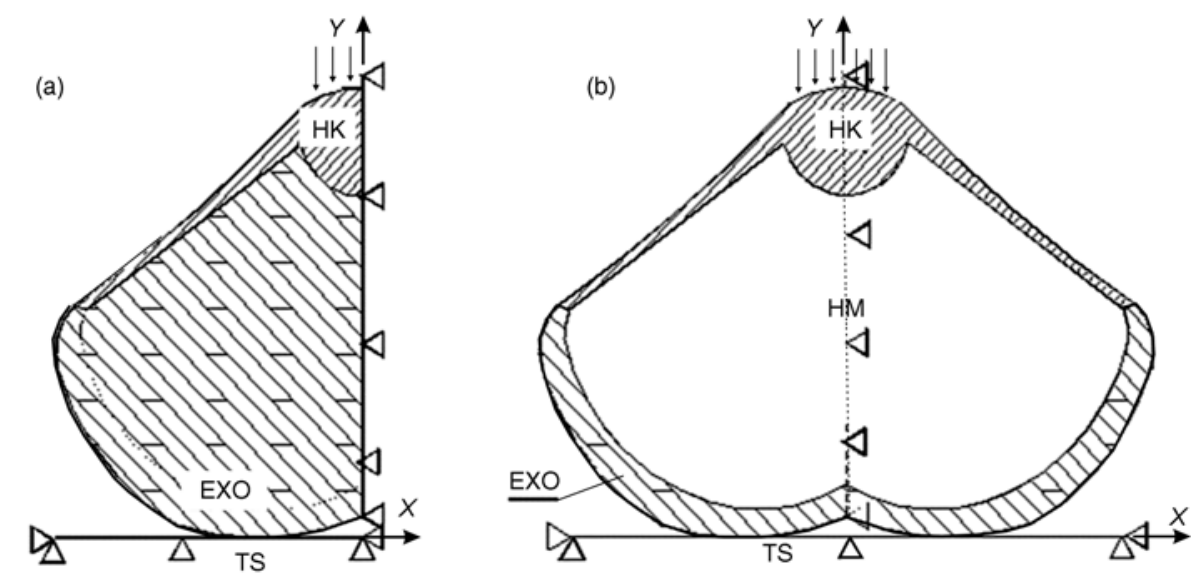

Figure 2 Finite element models. (a) Simplified model (SS), only two materials, soft excuticle EXO and Hard cuticle HK, are considered, TS Target surface, (b) fluid-contained model (FC), three materials, EXO, HK and hemolymph HM, are considered, fluid material HM in contained by EXO and HK. Target surface is rigid solid and stable-restrained in both $X$ and $Y$ directions. Symmetry axis $Y$ of both models is restrained in $X$ direction. 


\section{Results and discussions}

\subsection{Deformation}

Figures 3(a) and (b) show the deformation vectors of the grasshopper's attachment pad, Figures 3(c) and (d) are detailed images in the contact zone (marked by squares in (a) and (b)) for the SS and FC models respectively. The displacement vector fields were obtained under a restrained displacement of $0.1 \mathrm{~mm}$ at restrained $\mathrm{HK}$ surface. In order to show the deformation clearly, the displacement of each node points is magnified 10 times. For the SS model, the deformation vectors in the contact zone (Figure 3(a), square zone) are perpendicular to the target surface (Figure 3(c)), which suggests that no relative movement between pad and target surface is generated during the contact process, so it is reasonable to assume that no friction force is generated between the surfaces. On the other hand, the deformation vectors in the contact zone (Figure 3(b), square zone) in the FC model are parallel to the target surface (Figure 3(d)) and the directions of displacement vectors in two contact zones (Figure 3(b)) are reversed and are symmetrical in the $Y$ axis. So it is reasonable to assume that friction force in the reverse direction on contacted zones are created during the contact process and the frictional force increaseswith increasing loads. The generated friction forces, compared with that on the contact zones of SS model, would enhance the stability of the contact.
We believe that both geometric structure and the material topology make the displacement vector fields so different. The geometric design made it possible for the pads to move outside, but the tendency was more strongly restrained by the soft material (SS model) than that of fluid contained structures (FC model). This behavior results from the mechanical properties of two materials. As a fluid, hemolymph can bear only compressive stress, but not the shear stress and tensile stress. The compressive stress generated by the ground reaction force on contact zone and HK boundary was transmitted by hemolymph to the outside of container-like structure, leading to the displacement of node point in FC model in reversed directions (toward outside). On the contrary, the soft material can bear shear, tensile and compressive stresses, which restrains the motion of node point in SS model to move outside.

Knowledge of the function of tangential forces on animals' locomotion is fundamental to understanding their evolutionary development. Dickinson et al. ${ }^{[14]}$ examined the effects of ground-reaction force on the locomotion of animals, and pointed out that the groundreaction force on each foot or attachment pad is toward the body center. It has been demonstrated in gecko's setae that adhesion can be detected only when the setae slide on the target surface ${ }^{[23]}$. Our group developed several three-dimensional sensors and measured the ground reaction force of geckos (Gecko: Gecko), Erthesina Fullo Thunberg and Lycorma Delicatula White (Auche-

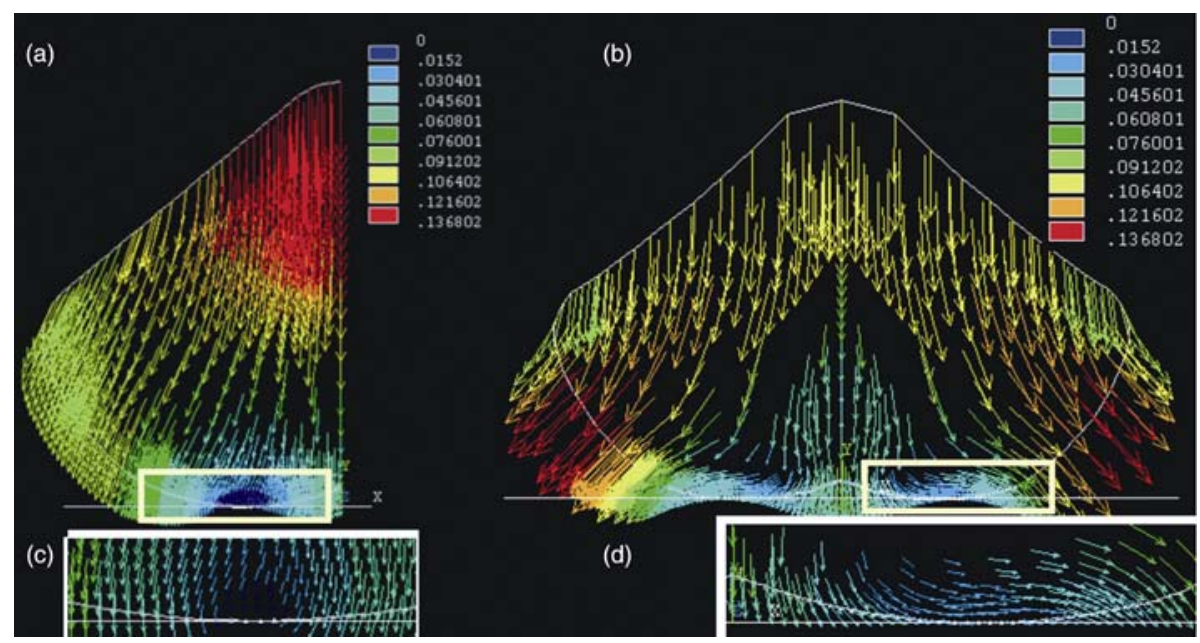

Figure 3 Vector field of displacements. (a) SS model and (c) detail of contact zone. The vectors in contact zone are perpendicular to the target surface, there is no relative movement between pad and target surface, so no friction force is generated during contact. (b) FC model and (d) detail of contact zone. The vectors in the contact zone are parallel to the target surface, a relative movement at reversed directions between pad and target surface is observed and thus reversed friction force will be generated during contact. The displacement vectors near contact zone in FC model ((b) and (d)) are much larger than those in SS model ((a) and (c)), suggesting that the increase rate of contact area in FC model is larger than in SS model. 
norrhyncha: Fulgoridae) on floor, wall and ceiling surfaces. All the data suggests that tangential force is always larger than the normal force when animals run on walls or ceilings, and the force is almost of the same order as the normal forces when they run on the floor. The relationships and functions of tangential force among several feet or pads are still not well understood.

Our analysis here reveals that the tangential force, generated between an attachment pad and the target surface, is redundant, which is conducive to enhancing the stability of the contact.

\subsection{Contact stiffness and contact area}

Figure 4 shows the relationship between displacement and load. The spring constants in the load direction are $K_{\mathrm{s}}=34.44 \mathrm{~N} / \mathrm{mm}$ and $K_{\mathrm{f}}=0.021 \mathrm{~N} / \mathrm{mm}$ for the SS model and FC models, respectively. The difference between the two models, $K_{\mathrm{s}} / K_{\mathrm{f}}$, is 1640 . This means that the geometric design and the material topology of a grasshopper's pad minimizes stiffness and makes the pad much flexible, even through the elastic modulus of the fluid contained in the area covered by EXO and HK (FC model) is much higher than that of EXO (SS model). Lower stiffness is useful for decreasing impact force during landing and prevents other parts of the leg from over being loaded. Big impact force is one of the major causes of failure in transmission gears in legged robots. This suggests that the geometric design of the grasshopper foot may be applicable to the design of legged robots as it may reduce impact forces.

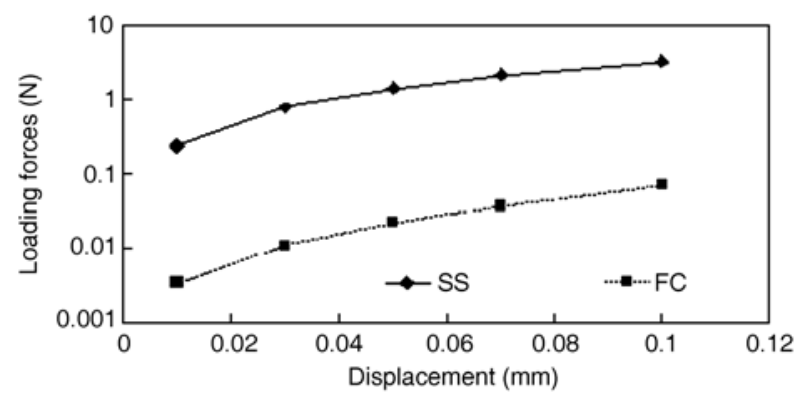

Figure 4 Reaction forces $F_{\mathrm{R}}$ for various pre-restrained displacement $D_{\mathrm{R}}$. Results show that $F_{\mathrm{R}}=34.41 \times D_{\mathrm{R}}-0.1851, n=5, r^{2}=$ 0.9969 for SS model and that $F_{\mathrm{R}}=0.7177 \times D_{\mathrm{R}}-0.009, n=5, r^{2}=$ 0.9613 for FC model. The ratio of contact spring constants is $R=$ $K_{\mathrm{s}} / K_{\mathrm{f}}=1640$, which means that geometric structure - the EXO and HK formed container filed with fluid hemolymph decrease the stiffness of the pads greatly.

Lower stiffness also means that the pad is more flexible, and larger contact area can be obtained during contact. Figure 5 shows the contact area for both SS and FC model, where the lengths of contacted lines were ob- tained by checking the reaction forces on the nodes of the contact elements. The zones were calculated by the length of contacted line when the reaction force on the node is not zero. Although the reaction force (namely the load acted) of FC model is smaller than that of SS model, the contacted line is much longer than that for the SS model, suggesting that in the fluid contained geometric design (FC model) it may be possible to increase adhesive force on the surface when grasshopper's attachment pads are attached on various surfaces, which is irrelevant to the adhesive mechanisms. Big real contact area always helps to obtain strong adhesive force. Jiao and Gorb ${ }^{[18]}$ has shown that adhesion in grasshoppers depended on the wet capillary force, which, again, depended on the contacting area. This analysis may answer why grasshopper could make their locomotion on up-side-down surfaces.

Our results suggests that in the geometric design of the grasshopper tarsal we may not only decrease landing impact force but also increase the contact area and thus
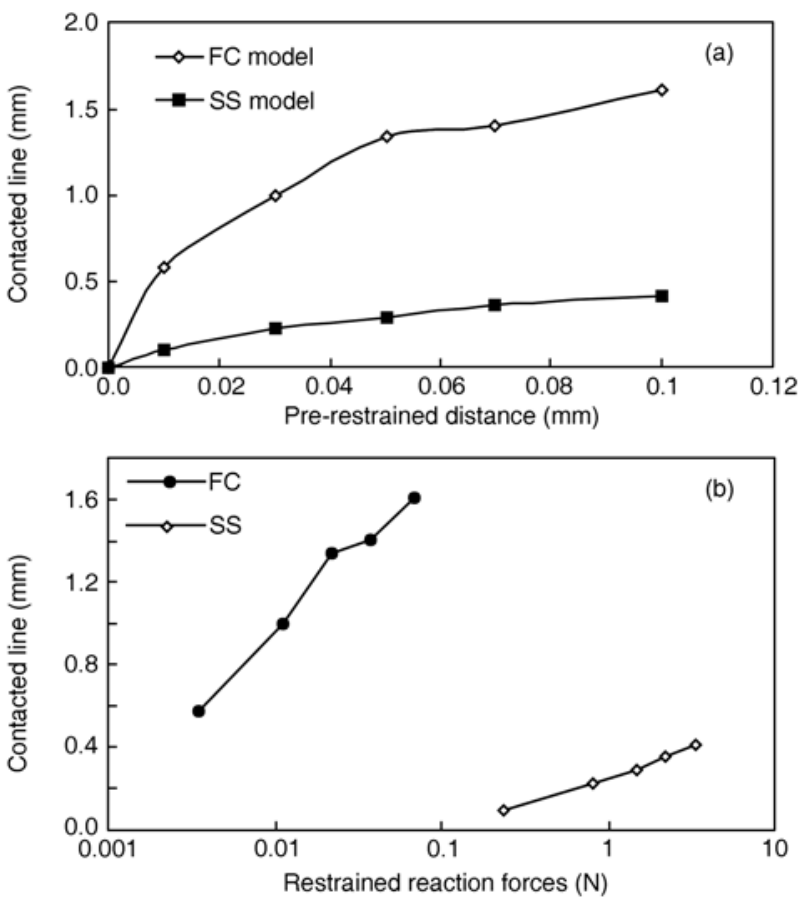

Figure 5 Relationship of contacted area with pre-restrained distance and restrained reaction forces. (a) Contact line Lc versus restrained displacement $\mathrm{Dr}$. Result regressions show that $L \mathrm{c}=$ $0.4508 \times \operatorname{Ln}(\mathrm{Dr})+2.6333, n=5, R^{2}=0.9882$ and $L c=0.1349 \times \operatorname{Ln}(\mathrm{Dr})$ $+0.7126, n=5, R^{2}=0.9888$ for FC and SS model respectively. (b) Contacted line $L c$ versus restrained reaction forces Frr. Results show that $L \mathrm{c}=0.3505 \times \operatorname{Ln}(\mathrm{Frr})+2.5839, R^{2}=0.9815$ for $\mathrm{FC}$ model and $L c=0.118 \times L n(F r r)+0.2619, R^{2}=0.991$ for SS model. These results mean that with the same restrained displacement, reaction forces of FC model are much lower than that of SS model, but the contact line are much higher than in SS model. 
increase the adhesion between grasshopper pads and target surface. Both of these characteristics are needed in designing three-dimensional obstacle-free robots.

\subsection{Effect of the airbag}

From the biological point of view, the function of airbag is still not clear. We suppose that the grasshopper could adjust the pressure in the airbag through "breath", namely decreasing the pressure to decrease the impact force in landing and increasing the pressure to minimize the adhesion in jumping. However it is still an open question whether grasshopper can actively control the contact stiffness in attachment pads by modulating the pressure in the airbag. From contact mechanics viewpoint, we have been puzzled for a long time on how to include the effect of airbag into the contact mechanics in FEA model. Since both air and hemolymph are fluids, the boundary between the two materials cannot be defined, so we integrated the airbag and hemolymph into a uniform liquid in our FC model to consider the effects of air pressure in airbag on the contact mechanics. We change the fluid's elastic modulus $E_{\mathrm{F}}$ from $3 \mathrm{GPa}$ to 12 $\mathrm{MPa}$ in EXO and HK surrounding area to simulate the effect of air pressure in airbag and the volume ratio of airbag to hemolymph. When the pressure modulation of the airbag is low, the integrated elastic modulus is also low (Figure 6). The results show that when the integrated modulus is lowered enough (lower than 60 $\mathrm{MPa}$ ), the stiffness is heavily decreased. This suggests the possibility that the grasshopper can control the contact status by modulating the pressure in the airbag. However it is still an open question whether the grasshopper can actively control the contact stiffness in attachment pads by modulating the pressure in the airbag.

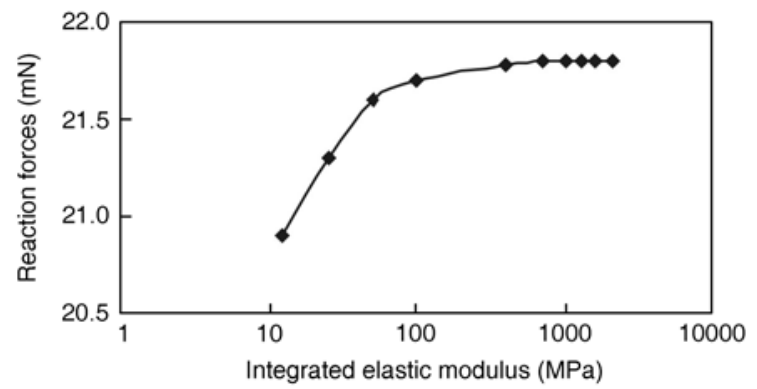

Figure 6 Reduction of integrated elastic modulus reduces the reaction forces for a given restrained displacement.

\subsection{Reaction forces on restrained points}

Figure 7 shows the reaction forces at restrained points in the $Y$ direction. The results show that with an increase in load (pre-restrained displacements), the reaction force also increases. The biggest reaction force is located in the HK zone and nearby, but in reverse direction, in the rod based tissue exocuticle that supports the superficial layer. The reaction force in the HM zone is lower than in the neighboring exocuticle. Interestingly the restrained force in the tendon area is zero. This result could explain why the tendon can keep its position in the hemolymph.

\subsection{Stress and strain of pads}

Figure 8 is an isoline image of the tensile stress parallel to the $X$-direction $\sigma_{x}$ when restrained displacement is 0.1 for both $\mathrm{SS}$ and FC models. Figure 8 shows that the

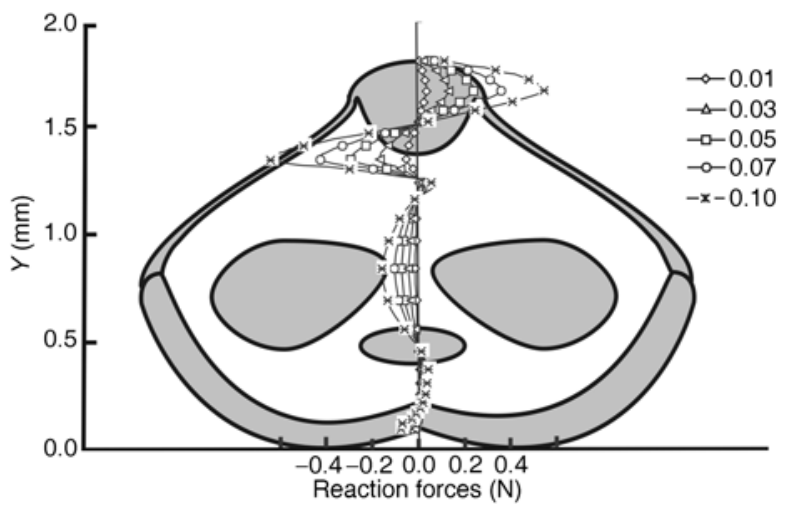

Figure 7 Reaction forces at restrained points for different preloads. With increasing loads, the restrained reaction forces are also increased. But the forces are always zero at the place where the flexor tendon is located.
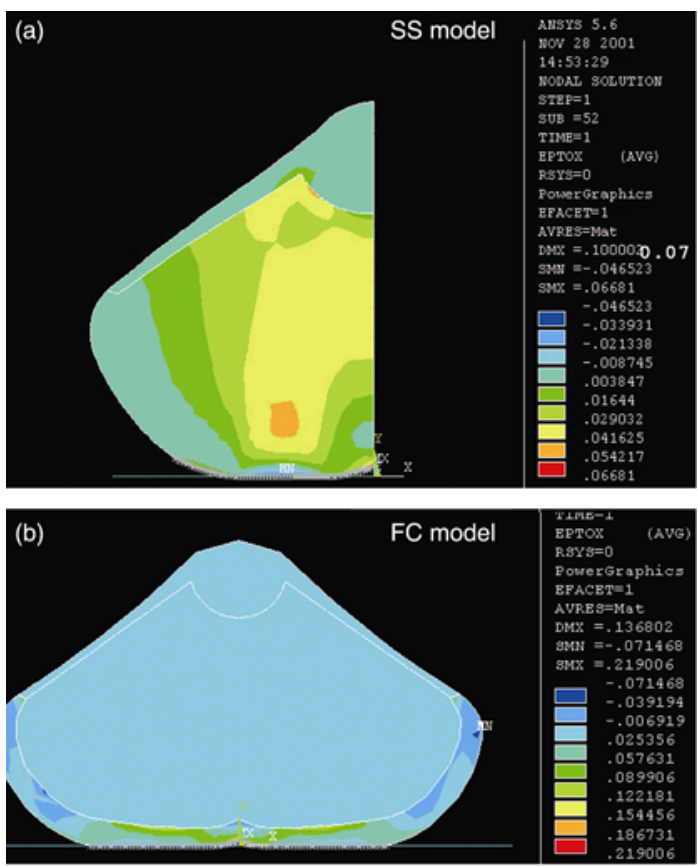

Figure 8 Tensile stress of grasshopper's pads in parallel direction to contact surface. 
maximum stress $\sigma_{x}$ for the SS model (Figure 8(a)) is located deeper than that for FC model (Figure 8(b)). The maximum tensile stress is $27 \mathrm{KPa}$ at a point $370 \mu \mathrm{m}$ from the contact surface for the SS model and $71.8 \mathrm{KPa}$ at the internal wall of the EXO for FC model.

\section{Conclusion}

The results obtained from the finite element analysis suggest that geometric evolution of grasshopper pads is

1 Dai Z D, Yu M, Ji A H, et al. Friction design of animal's driving pads and its bionics (in Chinese). Chin Mech Eng, 2005, 16(16): 1454-1457

2 Flannigan W C. Finite element modeling of arthropod exoskeleton. Thesis of master of sciences, Case Western Reserve University, May 1998

3 Cheung J T M, Zhang M, Leung A K L, et al. Three-dimensional finite element analysis of the foot during standing-a material sensitivity study. J Biomech, 2005, 38 (5): 1045-1054

4 Cheung J T M, Zhang M. A 3-dimensional finite element model of the human foot and ankle for insole design. Archi Phy Med Reha, 2005, 86(2): $353-358$

5 Cavanagh P R. Plantar soft tissue thickness during ground contact in walking. J Biomech, 1999, 32(6): 623-628

6 Dong X, Fan Y B, Zhang M, et al. Studies on Biomechanics of Human Foot: a Review. J Biom Engi, 2002, 19(1): 148-153

7 Gefen A, Megido-Ravid M, Itzchak Y, et al. Biomechanical analysis of the three-dimensional foot structure during gait: A basic tool for clinical applications. J Biom Engi-Trans ASME, 2000, 122(6): $630-639$

8 Gefen A, Linder-Ganz E. Diffusion of ulcers in the diabetic foot is promoted by stiffening of plantar muscular tissue under excessive bone compression (in German). Orthopade, 2004, 33(9): 999-1012

9 Richmond B G, Wright B W, Grosse L, et al. Finite element analysis in functional morphology. Anatomical record part A, 2005, 283A(2): $259-274$

10 Hanft J T, Hogan H A, Hood D M. Three-dimensional finite element modeling of the horse's foot. In: Proceedings of the 1995 fourteenth Biomedical Engineering Conference, 7-9, Apr. 1995, Shreveport, LA, USA, 59-62

11 Kesel A B, Philippi U, Nachtigall W. Biomechanical aspects of the insect wing: an analysis using the finite element method. Comp Biol Medi, 1998, 28: 423-437

12 Herbert R C, Young P G., Smith C W, et al. The hind wing of the desert locust (Schistocerca gregaria Forskal) III. A finite element analysis of a deployable structure. J Exp Biol, 2000, 203: 2945-2955

13 Wootton R J, Evans K E, Herbert R, et al. The hind wing of the desert locust (Schistocerca gregaria Forskal) I. Functional morphology and mode of operation. J Exp Biol, 2000, 203: 2921 - 2931

14 Dickinson M H, Farley C T, Full R J, et al. How animals move: an integrative view. Science, 2000, 288: 100-106

15 Nishikawa M, Kumagai T. 1990. Foot structure for legged walking optimized to increase contact area, reduce landing impact forces, and increase contact stability by generating reversed tangent force during contact formation.

The affects of elastic modulus on the contact parameters suggest that the grasshopper may have the ability to modulate contact status by control of the airbag pressure. For example, increasing the airbag pressure may make jumping easier while lowering the pressure during landing would ease impact force and increase adhesion when walking on inverted surfaces.

robot. EP 0433091A2

16 Berges P, Bowling A. Impact Forces in Legged Robot Locomotion. In: Proceedings of the 2005 IEEE International Conference on Robotics and Automation Barcelona, Spain, April 2005, 3745-3751

17 Gorb N S, Jiao Y K, Scherge M. Ultrastructureal architecture and mechanical properties of attachment pads in Tettigonia viridissima (Orthoptera Tettigoniidae). J Compa Phys, 2000, 186: 821 - 831

18 Jiao Y K, Gorb N S, Scherge M. Adhesion measured on the attachment pads of Tettigonia viridissima (Orthoptera Insecta). J Exp Biol, 2000, 203, 1887-1895

19 Goodwyn P P, Perssadko A, Schwart H, et al. Material structure, stiffness, and adhesion: why attachment pads of the grasshopper (Tettigonia viridissima) adhere more strongly than those of the grasshopper (Grasshoppera migratoia) (Insecta: Orthoptera). J Comp Physiol, 2006, 192: 1233-1243

20 Gorb N S, Scherge M. Biological microtribology: anisotropy in frictional forces of orthopteran attachment pads reflects the ultrastructure of a highly deformable materials, Proc. R. Soc. Lond, B 2000, 267: $1-7$

21 Stork N E. Experimental analysis of adhesive of Chrysolina polita (Chrysomelidae, Coleptera) on a variety of surfaces. J Exp Biol, 1980, 88: 583-597

22 Dixon A F G., Croghan P C, Gowing R P. The mechanism by which aphids adhere to smooth surface. J Exp Biol, 1999, 52: 243-253

23 Autumn K, Liang Y A, Hsieh S T, et al. Adhesive force of a single gecko foot-hair. Nature, 2000, 405: 681-685

24 Autumn K, Sitti M, Liang Y A, et al. Evidence for van der Waals adhesion in gecko setae. Proc Natl Acad Sci USA, 2002, 99, $12252-12256$

25 Gao H J, Wang X, Yao H M, et al. Mechanics of hierarchical adhesion structures of geckos. Mech Mater, 2005, 37: 275-285

26 Gillett J D, Wigglesworth V B. The climbing organ of an insect, Rhodnius prolixus (Hemiptera, Reduviidae). Proc R Sco Lond B 1932, 111: $364-376$

27 Maderson P F A. Keratinized epidermal derivatives as an aid to climbing in gekkonid lizards. Nature, 1964, 203: 780-781

28 Gabriel J M. The development of the locust jumping mechanism. Il energy storage and muscle mechanics. J Exp Biol, 1985, 118 $327-340$

29 Santer R D, Yamawaki Y, Rind F C, et al. Motor activity and trajectory control during escape jumping in the locust Locusta migratoria. J Comp Physiol A, 2005, 191: 965-975 